\title{
Simultaneous Determination of Four Compounds in a Nelumbo nucifera Seed Embryo by HPLC-DAD
}

\author{
Gahee Ryu, ${ }^{1}$ Jin Bae Weon, ${ }^{1}$ Woo Seung Yang, ${ }^{1}$ and Choong Je $\mathrm{Ma}^{1,2}$ \\ ${ }^{1}$ Department of Medical Biomaterials Engineering, College of Biomedical Science, Kangwon National University \\ Chuncheon 200-701, Republic of Korea \\ ${ }^{2}$ Institute of Bioscience and Biotechnology, Kangwon National University, Chuncheon 200-701, Republic of Korea \\ Correspondence should be addressed to Choong Je Ma; cjma@kangwon.ac.kr
}

Received 4 December 2016; Revised 19 January 2017; Accepted 2 February 2017; Published 13 April 2017

Academic Editor: K. S. V. Krishna Rao

Copyright (c) 2017 Gahee Ryu et al. This is an open access article distributed under the Creative Commons Attribution License, which permits unrestricted use, distribution, and reproduction in any medium, provided the original work is properly cited.

Nelumbo nucifera has a variety of biological activities. So it was importantly used as various herbal medicines since traditional times. A simple, fast, and sensitive high-performance liquid chromatographic (HPLC) method was developed in this study for efficient quality control of $N$. nucifera. Four different compounds, including neferine, 1,2,3,4-tetrahydro-1-[(4-hydroxyphenyl) methyl]-2-methyl-7-isoquinolinol, 1-hydroxy-2-methylpropene, and 3-(prop-1-enyl)benzene-1,2,4,5-tetrol, were simultaneously determined. The four compounds were isolated through a Dionex $\mathrm{C}_{18}$ column by gradient elution with $0.1 \%$ TFA-water and methanol. The flow rate was $1.0 \mathrm{~mL} / \mathrm{min}$, and the wavelength was detected at 205, 254, 280, and $330 \mathrm{~nm}$. The chromatograms were acquired at $205 \mathrm{~nm}$. The four compounds showed good linear relationships $\left(r^{2}>0.96\right)$ over five different concentrations, and an average recovery of the method ranged from $96.27 \%$ to $108.78 \%$. Through the analysis validation test and application of the method, the optimized conditions verified that it is efficient to isolate the compounds of $N$. nucifera seed embryos.

\section{Introduction}

Lotus (Nelumbo nucifera) is an aquatic plant, which is widely distributed in East Asia, Australia, and North America [1]. It is evaluated as one of the important Chinese herbal medicines. Nearly all of the parts of the lotus, including leaves, leaf stalks, flowers, seeds, and rhizomes, are edible and used as food products and medicines [2]. Different parts possess diverse activities such as anti-ischaemia, antioxidant, anticancer, hepatoprotection, antidiarrhoeal, and anti-inflammation.

The lotus seed is also known as "kamal gatta" in Indian markets, and its embryos are mainly used to treat nervous disorders, cardiovascular diseases, inflammation, and cancer [3]. N. nucifera is composed of many phenolic compounds. The major bioactive compounds of an N. nucifera seed are phenolic compounds, for example, lotusine, nuciferine, neferine, isoliensinine, and liensinie [4]. Phenolic compounds show high anti-inflammatory and antioxidant properties, which are determined by the presence of a hydroxyl group bonded to an aromatic ring to give the molecular formula, $\mathrm{C}_{6} \mathrm{H}_{5} \mathrm{OH}[5,6]$. Phenols have been isolated from various plants $[7,8]$, and recent studies have shown that the embryos of the lotus seed also have abundant phenolic compounds $[9,10]$. It has also been reported that seed embryos of the lotus have antioxidant activities [4]. Oxidative stress and immoderate inflammatory responses can be critical etiological causes of neurodegenerative diseases, for example, Alzheimer's disease, Parkinson's disease, and dementia [11]. Based on the relationship of the structure and the activity of antioxidant and anti-inflammation, a previous study that focused on the neuroprotective effect of lotus embryo had been accomplished before this report [12]. The study about an effective isolation method is also accomplished.

The purpose of this report is to establish a simple, sensitive, and fast HPLC analytical method for compounds of $N$. nucifera embryos. There are already some papers about the analysis of $N$. nucifera seed embryo compounds $[9,10]$, but in this report, the three compounds that have not been analyzed are simultaneously determined, including neferine. 
<smiles>COC(=O)N1CCc2cc(OC)c(Oc3cc(C[C@@H]4c5cc(OC)c(OC)cc5CCN4C)ccc3O)cc2[C@@H]1Cc1ccc(OC)cc1</smiles><smiles>[R]c1cc2c(cc1O)C(Cc1ccc(O)cc1)N(C)CC2</smiles>

1,2,3,4-Tetrahydro-1-[(4-hydroxyphenyl) methyl]2-methyl-7-isoquinolinol<smiles>CC(C=O)=CO</smiles>

1-Hydroxy-2-methylpropene

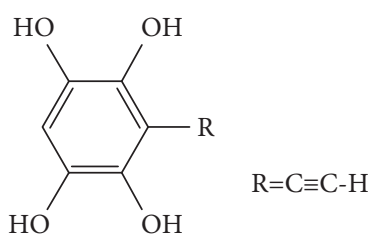

3-(Prop-1-enyl)benzene-1,2,4,5-tetrol

Figure 1: Chemical structures of compounds in N. nucifera embryos.

The described method will be useful for the quality control of this plant and the development of health food or medicine.

\section{Materials and Methods}

2.1. Plant Material. The N. nucifera seed embryos were supplied by the Wildlife Genetic Resources Center, National Institute of Biological Resources (Incheon, Korea). The materials were validated by Dr. Young Bae Seo, a professor at the College of Oriental Medicine, Daejeon University (Daejeon, Korea). The extracted and evaporated specimens (number CJ129M) were stored at Kangwon National University (Chuncheon, Korea).

2.2. Sample Preparation. The dried N. nucifera seed embryos $(15.75 \mathrm{~g})$ were extracted thrice with $80 \%$ methanol by ultrasonication in an ultrasonic bath at room temperature. The extracted samples were concentrated by a rotary evaporator and dissolved in water. Then, the water solution was partitioned in $\mathrm{n}$-hexane, $\mathrm{CHCl}_{3}$, and ethyl acetate (EtOAc). EtOAc fractions were isolated on a Sephadex LH-20 column and eluted once with $\mathrm{MeOH}$ : water $(4: 1)$. Three compounds, 1,2,3,4-tetrahydro-1-[(4-hydroxyphenyl) methyl]-2-methyl7-isoquinolinol, 1-hydroxy-2-methylpropene, and 3-(prop1-enyl)benzene-1,2,4,5-tetrol, were separated using HPLC on a YMC C18 $(250 \mathrm{~mm} \times 10 \mathrm{~mm}$ I.D. S- $5 \mu \mathrm{m})$ column and a LUNA $(250 \mathrm{~mm} \times 10 \mathrm{~mm}$ I.D. S- $5 \mu \mathrm{m})$ column. The chemical structures of compounds are shown in Figure 1. Neferine was purchased from Sigma-Aldrich (USA). HPLC grade solvents, water, and methanol were purchased from J.T. Baker (USA). TFA (trifluoroacetic acid) was purchased from DAE JUNG in Korea.
2.3. Preparation of Standard Solutions. Standard stock solutions were produced by dissolving neferine, 1,2,3,4tetrahydro-1-[(4-hydroxyphenyl) methyl]-2-methyl-7-isoquinolinol, 1-hydroxy-2-methylpropene, and 3-(prop-1enyl)benzene-1,2,4,5-tetrol in $80 \%$ methanol at a concentration of $362.50 \mu \mathrm{g} / \mathrm{mL}, 404.16 \mu \mathrm{g} / \mathrm{mL}, 362.50 \mu \mathrm{g} / \mathrm{mL}$, and $387.50 \mu \mathrm{g} / \mathrm{mL}$, respectively. $1 / 2,1 / 4,1 / 8$, and $1 / 16$ times diluted working solutions were prepared by dissolving each standard stock solution in $80 \% \mathrm{MeOH}$. Diluted samples were filtered through a $0.45 \mu \mathrm{m}$ pore-size filter. The manufactured working solutions were used to establish a calibration curve.

2.4. HPLC Analysis. All the solvents were HPLC grade and purchased from J.T. Baker. N. nucifera solutions were analyzed by HPLC-DAD. The high-performance liquid chromatography (HPLC) (Dionex) was composed of an LPG $3 X 00$ pump, an ACC-3000 autosampler, a DAD-3000(RS) diode array UV/VIS detector, and a column oven. Each sample was injected and isolated through a Dionex $\mathrm{C}_{18}$ column $(5 \mu \mathrm{m}, 120 \AA ., 4.6 \mathrm{~mm} \times 150 \mathrm{~mm})$ at $25^{\circ} \mathrm{C}$. The mobile phase consisted of $0.1 \%$ TFA-water and methanol. The injection volume of samples was $20 \mu \mathrm{L}$. The output signal of the detector was transcribed by a Dionex Chromeleon ${ }^{\mathrm{TM}}$ Chromatography Data System. The UV wavelength was 205, 254,280 , and $330 \mathrm{~nm}$, respectively, and the chromatograms were acquired at $205 \mathrm{~nm}$.

\subsection{Validation Method}

2.5.1. Calibration Curve. The calibration curves were built by plotting the peak area versus concentration of each working solution. To prepare the working solution, neferine $(22.66,45.31,90.63,181.25$, and $362.50 \mu \mathrm{g} / \mathrm{mL})$, 1,2,3,4-tetrahydro-1-[(4-hydroxyphenyl) methyl]-2-methyl- 


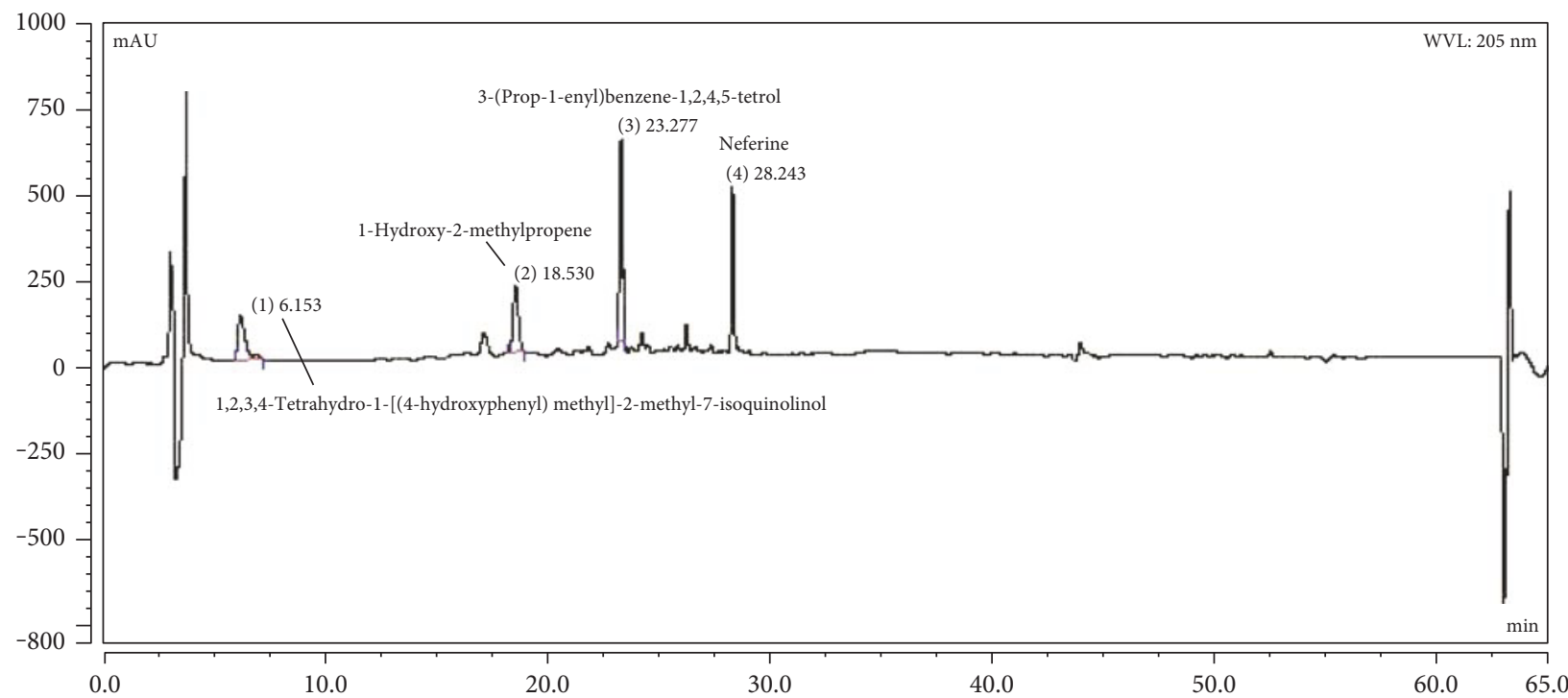

(a)

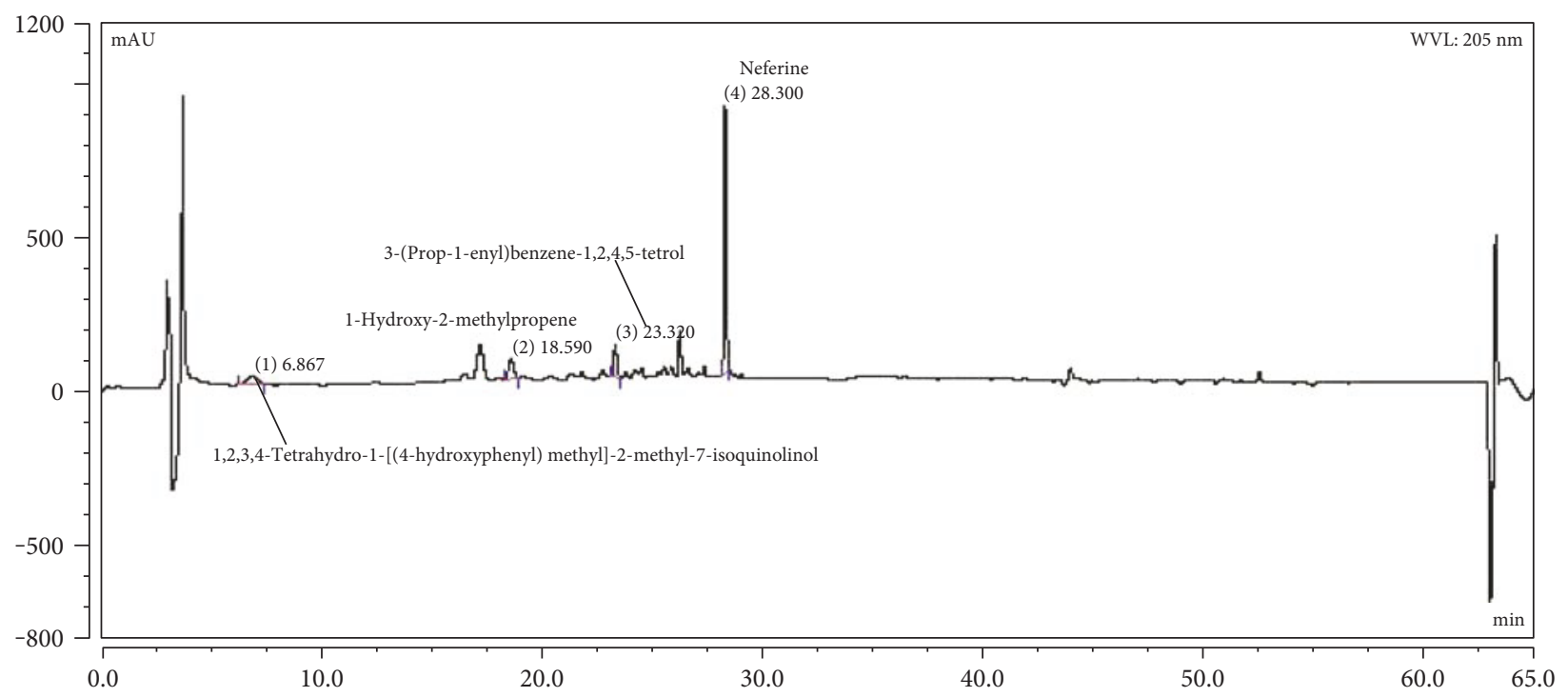

(b)

Figure 2: The HPLC-DAD chromatograms of standard compounds (a) and compounds from N. nucifera embryos extracted with $80 \%$ methanol (b). 1,2,3,4-Tetrahydro-1-[(4-hydroxyphenyl) methyl]-2-methyl-7-isoquinolinol (1), 1-hydroxy-2-methylpropene (2), 3-(prop-1enyl)benzene-1,2,4,5-tetrol (3), and neferine (4).

7-isoquinolinol (25.26, 50.52, 101.04, 202.08, and $404.16 \mu \mathrm{g} / \mathrm{mL}), 1$-hydroxy-2-methylpropene (22.66, 45.31, $90.63,181.25$, and $362.50 \mu \mathrm{g} / \mathrm{mL}$ ), and 3-(prop-1-enyl)benzene-1,2,4,5-tetrol (24.22, 48.44, 96.88, 193.75, and $387.50 \mu \mathrm{g} / \mathrm{mL}$ ) were dissolved in methanol. The linear regression equations were calculated by $y=a x \pm b$, where $x$ and $y$ are the concentrations and the peak areas of each flavonoid, respectively. The linearity was established according to the least squares treatment $\left(R^{2}\right)$. Analysis of each working standard concentration was done in triplicate.

2.5.2. Limit of Detection (LOD) and Limit of Quantification (LOQ). The limit of detection (LOD) was determined as the lowest concentration of sample, and the limit of detection quantification (LOQ) was determined as the lowest concentration of compounds by injecting the diluted standard solution when the signal-to-noise ratio reached 3.3 10.

2.5.3. Repeatability and Precision. The precisions of the method were evaluated by intraday and interday injections. The intraday experiment was performed by three injections for a day, and the interday was performed by three injections for 3 days. Both tests were examined in three different concentrations which were confirmed in calibration curves. The reproducibility and precision were expressed as relative standard deviations (RSD, \%).

2.5.4. Recovery Test. The accuracy of the method was evaluated by a recovery study. It was conducted by adding a measured amount of compound solutions to the whole $N$. 
nucifera sample. Three different concentrations of neferine (45.31, 90.63, and $181.25 \mu \mathrm{g} / \mathrm{mL})$, ethanone $(50.52,101.04$, and $202.08 \mu \mathrm{g} / \mathrm{mL}$ ), isoquinolinol $(45.31,90.63$, and $181.25 \mu \mathrm{g} / \mathrm{mL}$ ), and isoquinolinediol $(48.44,96.88$, and $193.75 \mu \mathrm{g} / \mathrm{mL}$ ) were added to the samples. The equation used to define the recovery percentage was (detected amount - original amount)/spiked amount $\times 100$.

2.6. Sample Analysis Using Established Method. The efficiency of optimized simultaneous determination method was verified by analyzing the total N. nucifera sample. Each content of four target compounds was detected and evaluated.

\section{Results and Discussions}

Four phenolic compounds in the lotus embryo, including neferine, 1,2,3,4-tetrahydro-1-[(4-hydroxyphenyl) methyl]2-methyl-7-isoquinolinol, 1-hydroxy-2-methylpropene, and 3-(prop-1-enyl)benzene-1,2,4,5-tetrol, were identified by nuclear magnetic resonance (NMR) spectroscopy and their chemical structures were investigated. All compounds were isolated and analyzed by HPLC-DAD.

3.1. Optimization of HPLC-DAD Conditions. The reason for an optimized experiment using HPLC was to gain higher separation efficiency and peak resolution of target compounds in a shorter run time. To achieve this goal in Nelumbo nucifera, the effective HPLC-DAD conditions including suitable column, mobile phase, and wavelength of UV spectrum were chosen by performing many preliminary tests. The selected column was a Dionex $\mathrm{C}_{18}$ column, one of reversed-phase columns. The multistep gradient solution system consisted of $0.1 \%$ TFA-water (A) and methanol (B) was used as a mobile phase. The most appropriate condition for the separation of seed embryo was $0-10 \mathrm{~min}, 10 \% \mathrm{~B} ; 10-20 \mathrm{~min}, 10-20 \% \mathrm{~B}$; $20-25 \mathrm{~min}, 20-30 \% \mathrm{~B}$; and $25-30 \mathrm{~min}, 30 \% \mathrm{~B}$ at a flow rate of $1.0 \mathrm{~mL} / \mathrm{min}$. This gradient led to the separation of four peaks within $30 \mathrm{~min}$. The wavelength of the DAD detector was tested at 205, 254, 280, and $330 \mathrm{~nm}$ and the chromatograms were selected at $205 \mathrm{~nm}$ by UV spectrum. The column temperature had no significant influence on the separation, so room temperature was used. The peak of each compound was assured by comparing retention time and UV spectrum of their corresponding marker solution. The chromatograms of the N. nucifera compounds are shown in Figure 2.

3.2. Analysis Method Validation. To approve the validation of this method, the experiments for verifying linearity, detection and quantification limits, precision and accuracy, and recovery were performed. The regression equations were established by five concentration trials of each standard in triplicate. In linear regression, equation $Y=a x+b$, where $x$ is a concentration of marker components, and $Y$ is the peak area. The slope and intercept of the calibration curve were determined by this equation. The high correlation coefficients $\left(r^{2}>0.96\right)$ showed that all calibration curves had good linearities within the test ranges as shown in Table 1. According to this linear regression, the results of the limits of detection (LOD) ranged from 1.31 to $4.4 \mu \mathrm{g} / \mathrm{mL}$ and limits of quantification (LOQ) were in the range of 3.98-
TABLE 1: Calibration curves, LOD, and LOQ of four compounds in N. nucifera embryos. Neferine (1), 1,2,3,4-tetrahydro-1-[(4hydroxyphenyl) methyl]-2-methyl-7-isoquinolinol (2), 1-hydroxy2-methylpropene (3), and 3-(prop-1-enyl)benzene-1,2,4,5-tetrol (4).

\begin{tabular}{lcccc}
\hline Compounds & Regression equation & $R^{2}(n=5)$ & $\begin{array}{c}\text { LOD } \\
(\mu \mathrm{g} / \mathrm{mL})\end{array}$ & $\begin{array}{c}\text { LOQ } \\
(\mu \mathrm{g} / \mathrm{mL})\end{array}$ \\
\hline 1 & $y=1.351 x+36.68$ & 0.962 & 1.3120 & 3.9758 \\
2 & $y=0.209 x+0.376$ & 0.999 & 4.3690 & 13.2394 \\
3 & $y=0.247 x+0.108$ & 1 & 2.2691 & 6.8759 \\
4 & $y=0.441 x+0.043$ & 1 & 1.8396 & 5.5747 \\
\hline
\end{tabular}

TABLE 2: Intra- and interday precision of four compounds in the extract of lotus embryo by HPLC-DAD. Neferine (1), 1,2,3,4-tetrahydro-1-[(4-hydroxyphenyl) methyl]-2-methyl-7isoquinolinol (2), 1-hydroxy-2-methylpropene (3), and 3-(prop-1enyl)benzene-1,2,4,5-tetrol (4).

\begin{tabular}{cccccc}
\hline \multirow{2}{*}{ Compounds $\begin{array}{c}\text { Concentrations } \\
(\mu \mathrm{g} / \mathrm{mL})\end{array}$} & $\begin{array}{c}\text { Intraday }(n=3) \\
\text { Mean } \\
(\mu \mathrm{g} / \mathrm{mL})\end{array}$ & $\begin{array}{c}\text { RSD } \\
(\%)\end{array}$ & $\begin{array}{c}\text { Mean } \\
(\mu \mathrm{g} / \mathrm{mL})\end{array}$ & $\begin{array}{c}(n=3) \\
\mathrm{RSD}^{\mathrm{a}}\end{array}$ \\
\hline \multirow{3}{*}{1} & 362.5 & 351.97 & 0.30 & 359.77 & 6.08 \\
& 181.25 & 225.79 & 0.53 & 222.35 & 0.87 \\
& 90.63 & 103.69 & 0.58 & 103.86 & 1.81 \\
\hline \multirow{3}{*}{3} & 404.16 & 411.85 & 0.48 & 411.73 & 0.05 \\
& 202.08 & 205.06 & 0.56 & 205.02 & 0.34 \\
& 101.04 & 101.50 & 1.04 & 101.52 & 0.54 \\
\hline & 362.5 & 364.57 & 0.59 & 364.22 & 0.50 \\
4 & 181.25 & 183.53 & 1.35 & 181.32 & 0.90 \\
& 90.63 & 90.82 & 0.78 & 89.99 & 1.54 \\
\hline & 387.5 & 388.06 & 0.23 & 388.83 & 0.40 \\
& 193.75 & 193.93 & 0.44 & 193.49 & 0.26 \\
& 96.88 & 96.86 & 0.57 & 96.96 & 0.14 \\
\hline
\end{tabular}

${ }^{\mathrm{a}} \mathrm{RSD}$ : relative standard deviation.

$13.24 \mu \mathrm{g} / \mathrm{mL}$, respectively. This means that a few compounds can be detected and quantified.

To evaluate the reproducibility and precision of the method, within-day test (intraday analysis, $n=3$ ) and intermediate-day test (interday analysis, $n=3$ ) were performed. The intraday test was examined three times during a single day, and the interday test was examined on three different days ( 1,3 , and 5 days). The RSD values of the intraday test were $0.23 \%-1.35 \%$ and interday were $0.05 \%-6.08 \%$, respectively (Table 2). These results showed that the method was very reproducible and precise.

The accuracy of the method was evaluated by carrying out a recovery test. Each sample was tested in triplicate by adding three different concentrations of solutions to the total $N$. nucifera standard solution. The mean recovery was $105.49 \%$ for neferine, $98.07 \%$ for 1,2,3,4-tetrahydro-1-[(4hydroxyphenyl) methyl]-2-methyl-7-isoquinolinol, 100.98\% for 1-hydroxy-2-methylpropene, and $102.15 \%$ for 3-(prop1-enyl)benzene-1,2,4,5-tetrol (Table 3). The accuracy of the methods was determined by the relative standard deviation (RSD). The \% RSD of the average recovery was $1.13 \%$ for 
TABle 3: Recoveries of 4 compounds in an N. nucifera embryo. Neferine (1), 1,2,3,4-tetrahydro-1-[(4-hydroxyphenyl) methyl]-2methyl-7-isoquinolinol (2), 1-hydroxy-2-methylpropene (3), and 3-(prop-1-enyl)benzene-1,2,4,5-tetrol (4).

\begin{tabular}{ccccc}
\hline Compounds & $\begin{array}{c}\text { Spiked } \\
\text { amount }(\mu \mathrm{g})\end{array}$ & $\begin{array}{c}\text { Measured } \\
\text { amount }(\mu \mathrm{g})\end{array}$ & $\begin{array}{c}\text { RSD } \\
(\%)\end{array}$ & $\begin{array}{c}\text { Recovery }^{\mathrm{a}} \\
(\%)\end{array}$ \\
\hline \multirow{4}{*}{180.18} & 181.25 & 1.74 & 99.41 \\
& 98.59 & 90.63 & 0.53 & 108.78 \\
2 & 49.06 & 45.31 & 1.12 & 108.27 \\
\hline \multirow{3}{*}{3} & 194.54 & 202.08 & 1.03 & 96.27 \\
& 98.13 & 101.04 & 4.66 & 97.12 \\
& 50.93 & 50.52 & 1.55 & 100.81 \\
\hline & 180.15 & 181.25 & 0.65 & 99.39 \\
4 & 93.06 & 90.63 & 2.40 & 102.68 \\
& 45.70 & 45.31 & 2.12 & 100.86 \\
\hline
\end{tabular}

${ }^{\mathrm{a}}$ Recovery $(\%)=($ observed amount - original amount $) /$ spiked amount $\times 100 \%$.

neferine, 2.41\% for 1,2,3,4-tetrahydro-1-[(4-hydroxyphenyl) methyl]-2-methyl-7-isoquinolinol, $1.72 \%$ for 1-hydroxy-2methylpropene, and $0.79 \%$ for 3-(prop-1-enyl)benzene1,2,4,5-tetrol. All the analyzed compounds were in the ideal range of recovery and RSDs were also low. This data shows the high accuracy of the method.

All the previously mentioned results indicate that the method was very suitable for the quantitative analysis of $N$. nucifera embryo samples.

3.3. Sample Analysis Using an Established Method. The method described above was successfully applied to investigate the four target compounds' content in $N$. nucifera seed embryos. As summarized in Table 4, the amounts of the tested compounds varied significantly. The dominant content of the compound was neferine which was $79.39 \mathrm{mg} / \mathrm{g}$ in the whole sample. Other compounds were relatively small in quantity. The content of 1,2,3,4-tetrahydro-1-[(4-hydroxyphenyl) methyl]-2-methyl-7-isoquinolinol, 1-hydroxy-2methylpropene, and 3-(prop-1-enyl)benzene-1,2,4,5-tetrol were $9.56 \mathrm{mg}, 13.83 \mathrm{mg}$, and $6.75 \mathrm{mg}$ per $1 \mathrm{~g}$ of $N$. nucifera sample, respectively. A total of four compounds were able to be detected simultaneously by HPLC-DAD in optimized conditions.

\section{Conclusion}

In this study, the developed HPLC-DAD method was used for the qualitative analysis of compounds in Nelumbo nucifera seed embryos. Four compounds, including neferine, 1,2,3,4-tetrahydro-1-[(4-hydroxyphenyl) methyl]-2-methyl7-isoquinolinol, 1-hydroxy-2-methylpropene, and 3-(prop1-enyl)benzene-1,2,4,5-tetrol, were simultaneously detected within $30 \mathrm{~min}$ for the first time. Also, the optimized method was selective and accurate which was verified by the validation test including linearity, limits of detection and
TABle 4: Contents $(\mu \mathrm{g} / \mathrm{g})$ of 4 compounds in $N$. nucifera embryo samples $(n=3)$. Neferine (1), 1,2,3,4-tetrahydro-1-[(4hydroxyphenyl) methyl]-2-methyl-7-isoquinolinol (2), 1-hydroxy2-methylpropene (3), and 3-(prop-1-enyl)benzene-1,2,4,5-tetrol (4).

\begin{tabular}{lcccc}
\hline Compounds & 1 & 2 & 3 & 4 \\
Contents $(\mu \mathrm{g} / \mathrm{g})$ & 79.39 & 9.56 & 13.83 & 6.75 \\
\hline
\end{tabular}

quantification, repeatability, precision, and accuracy test. These qualitative and quantitative analytical results can help with the quality control of N. nucifera seed embryos. According to a previous study, neferine showed significant neuroprotective effects, among the four compounds $[12,13]$. Based on the activity of the compounds, this method is useful to develop the healthy food or medicine for neuroprotection. It is also expected to be used in research and development of activities and biological mechanisms.

\section{Conflicts of Interest}

The authors declare that they have no conflicts of interest.

\section{Acknowledgments}

This work was supported by the National Research Foundation of Korea (NRF) grant funded by the Korean government (Ministry of Science, ICT \& Future Planning) (no. 2016R1A2B1011384).

\section{References}

[1] S. Chen, B. H. Wu, J. B. Fang et al., "Analysis of flavonoids from lotus (Nelumbo nucifera) leaves using high performance liquid chromatography/photodiode array detector tandem electrospray ionization mass spectrometry and an extraction method optimized by orthogonal design," Journal of Chromatography A, vol. 1227, pp. 145-153, 2012a.

[2] S. Chen, L. Fang, H. Xi et al., "Simultaneous qualitative assessment and quantitative analysis of flavonoids in various tissues of lotus (Nelumbo nucifera) using high performance liquid chromatography coupled with triple quad mass spectrometry," Analytica Chimica Acta, vol. 724, pp. 127-135, 2012 b.

[3] P. K. Mukherjee, D. Mukherjee, A. K. Maji, S. Rai, and M. Heinrich, "The sacred lotus (Nelumbo nucifera)-phytochemical and therapeutic profile," Journal of Pharmacy and Pharmacology, vol. 61, no. 4, pp. 407-422, 2009.

[4] S. Rai, A. Wahile, K. Mukherjee, B. P. Saha, and P. K. Mukherjee, "Antioxidant activity of Nelumbo nucifera (sacred lotus) seeds," Journal of Ethnopharmacology, vol. 104, no. 3, pp. 322-327, 2006.

[5] C. V. Rao, A. Rivenson, B. Simi, and B. S. Reddy, "Chemoprevention of colon carcinogenesis by dietary curcumin, a naturally occurring plant phenolic compound," Cancer Research, vol. 55, no. 2, pp. 259-266, 1995.

[6] C. Li, H. Du, L. Wang et al., "Flavonoid composition and antioxidant activity of tree peony (Paeonia section Moutan) yellow flowers," Journal of Agricultural and Food Chemistry, vol. 57, no. 18, pp. 8496-8503, 2009.

[7] Y. Cai, Q. Luo, M. Sun, and H. Corke, "Antioxidant activity and phenolic compounds of 112 traditional Chinese medicinal 
plants associated with anticancer," Life Sciences, vol. 74, no. 17, pp. 2157-2184, 2004.

[8] M. P. Kähkönen, A. I. Hopia, H. J. Vuorela et al., "Antioxidant activity of plant extracts containing phenolic compounds," Journal of Agricultural and Food Chemistry, vol. 47, no. 10, pp. 3954-3962, 1999.

[9] Y. Chen, G. Fan, H. Wu, Y. Wu, and A. Mitchell, "Separation, identification and rapid determination of liensine, isoliensinine and neferine from the embryo of the seed of Nelumbo nucifera Gaertn. by liquid chromatography coupled to diode array detector and tandem mass spectrometry," Journal of Pharmaceutical and Biomedical Analysis, vol. 43, no. 1, pp. 99-104, 2007.

[10] S. Wu, C. Sun, X. H. Cao, H. Zhou, Z. Hong, and Y. Pan, "Preparative counter-current chromatography isolation of liensinine and its analogues from the embryo of the seed of Nelumbo nucifera GAERTN. using upright coil planet centrifuge with four multilayer coils connected in series," Journal of Chromatography A, vol. 1041, no. 1, pp. 153-162, 2004.

[11] N. Cho, J. H. Choi, H. Yang et al., "Neuroprotective and antiinflammatory effects of flavonoids isolated from Rhus verniciflua in neuronal HT22 and microglial BV2 cell lines," Food and Chemical Toxicology, vol. 50, no. 6, pp. 1940-1945, 2012.

[12] E. S. Kim, J. B. Weon, B. R. Yun et al., "Cognitive enhancing and neuroprotective effect of the embryo of the Nelumbo nucifera seed," Evidence-based Complementary and Alternative Medicine, vol. 2014, article 869831, p. 9, 2014.

[13] H. A. Jung, S. Karki, J. H. Kim, and J. S. Choi, "BACE1 and cholinesterase inhibitory activities of Nelumbo nucifera embryos," Archives of Pharmacal Research, vol. 38, no. 6, pp. 1178-1187, 2015. 

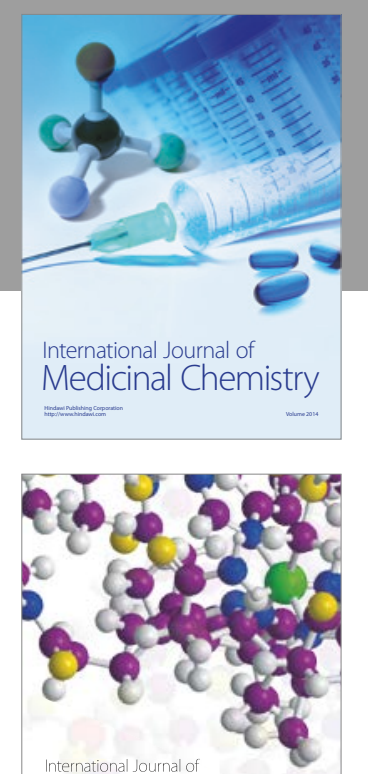

Carbohydrate Chemistry

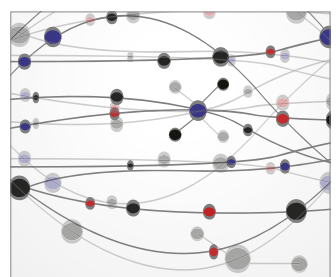

The Scientific World Journal
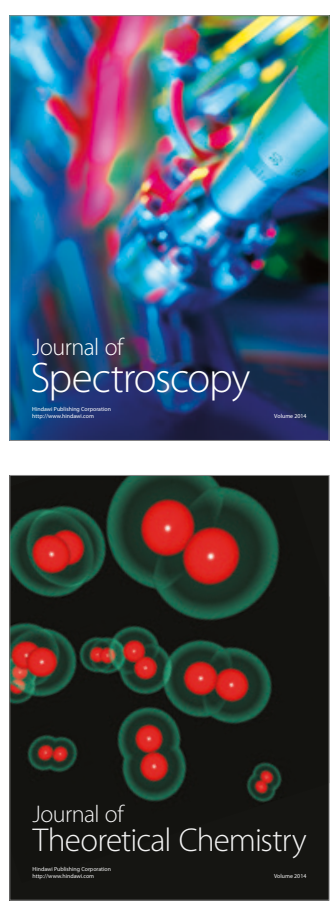
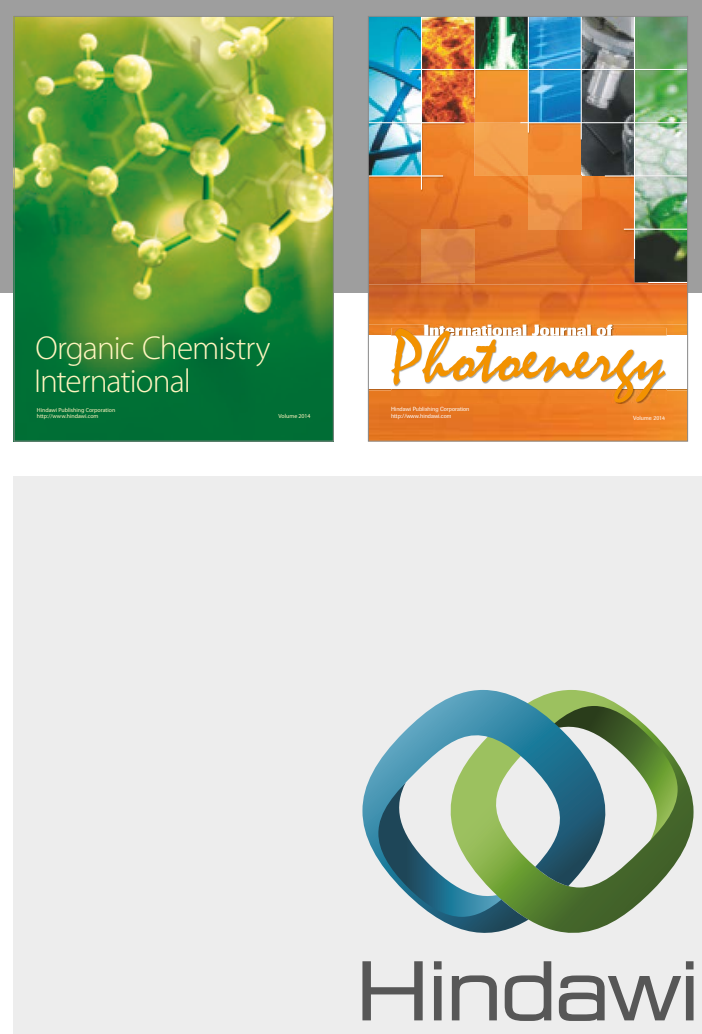

Submit your manuscripts at

https://www.hindawi.com

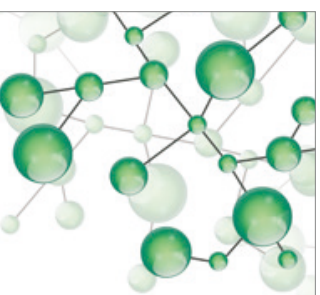

International Journal of

Inorganic Chemistry

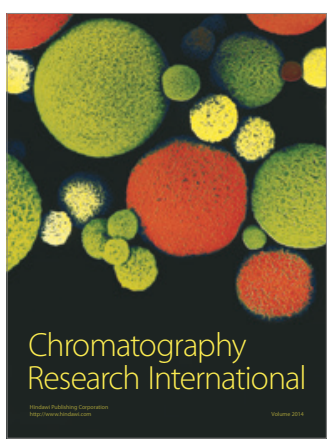

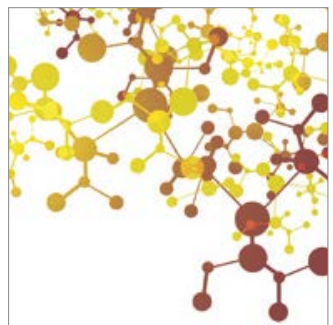

Applied Chemistry
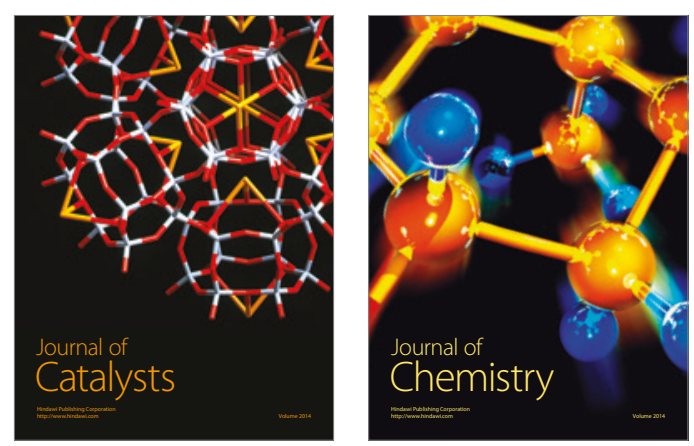
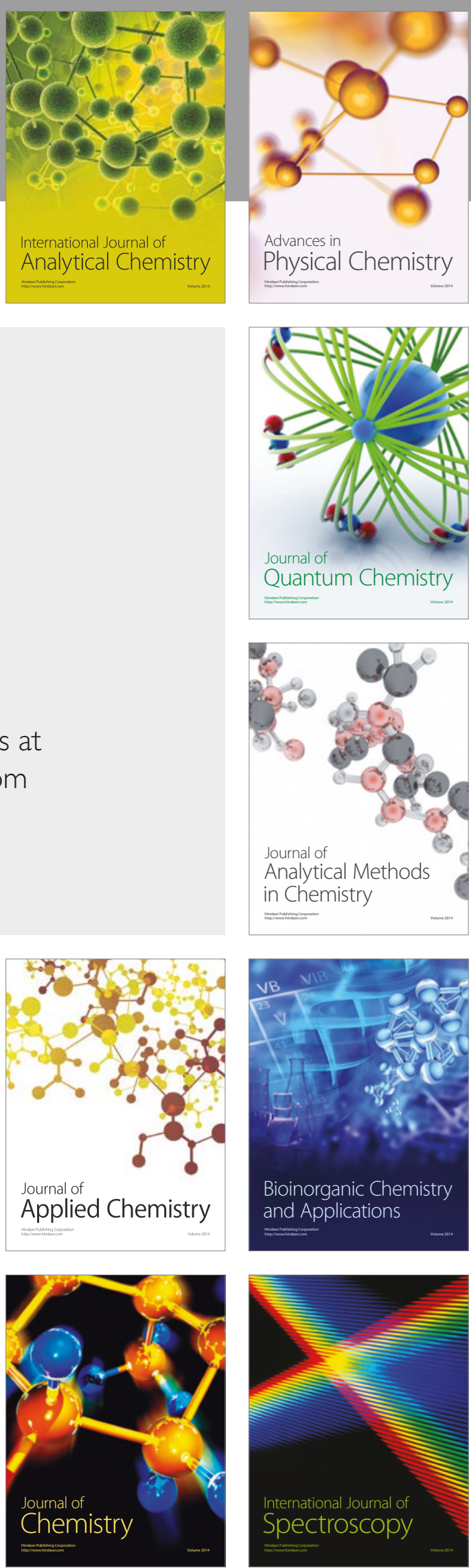